\title{
Design and Implementation of Online Data Assessment Based on E Format
}

\author{
Tao Liu ${ }^{\mathrm{a}}$, Tao $\mathrm{Wu}^{\mathrm{b}} *$, Jian Qiu ${ }^{\mathrm{c}}$, Guoqiang $\mathrm{Li}^{\mathrm{b}}$ \\ ${ }^{a}$ Center China Grid Company Limited, Wuhan, 430077,China \\ ${ }^{b}$ North China Electric Power University, Beijing, 102206,China \\ ${ }^{c}$ China Electric Power Research Institute, Beijing, 100192,China
}

\begin{abstract}
With the extended development of complication of power system, the accurate reflection of power system operation has become an important factor of power system stability. In order to get more accurate data, this paper proposes a software design philosophy of online data assessment system based on E format, which includes Online/Offline data modeling matching, online/offline parameter estimation and online data adjustment. The research plays an important role on practical power system real-time analysis.
\end{abstract}

Keywords: E format, online/offline data modeling match, online data adjustment, online offline parameter estimation.

\section{Introduction}

As an important industry of the national economy, electric power industry plays an irreplaceable role on the country's sustainable development and people's normal lives and work. With the trend of interconnection and high-voltage on power system in recent years, the increase of the scale of power grid is also leading to a grid of the serious accident. At the end of last century, the occurrence of many breakdowns on power system worldwide brought momentous influence. The USA Canada blackout on $14^{\text {th }}$, Aug, 2003, involved more than 50 million people, load loss as high as $61.8 \mathrm{GW}$, and economic loss estimated to be 4-10 billion [1-3]. A series of power outage also happen in China, such as Hainan power grid blackout accidents on $26^{\text {th }}$, Sep, 2006, Tibet power system blackout accident on $24^{\text {th }}$, Oct, 2005, Henan power grid blackout accidents on $1^{\text {st }}$, Jul, 2006. Most of the accidents occurred are due to lacking of effective monitoring and warning system, failing control on real-time stability of the power grid and taking ineffective control measures [4]. A much higher level of power system dispatching is in need in current smart grid construction. At present, online dynamic security evaluation system and power system warning system has been put into use in many provinces' power system dispatching centers. The system has an important meaning on optimizing the power system structure, improving the transmission ability and the quality of power supply. The whole online dynamic safety assessment system based on E language format acts as a carrier, which takes data integration between collected online operation data and offline analysis calculation data, and then adopts the data integration for analysis of online stability, voltage stability, small signal stability and etc. [5]. The integrated data can not only reflects the information of power system real-time operation status, but also contains the detailed parameters of entire power system model and equipment. However, there are still some problems about data entry, parameter management, parameter estimation, mapping processing, which results in lower level of accuracy of the online data calculating. Therefore, high-quality data sources and fast online offline estimation algorithm is the key to online dynamic security assessment and warning systems.

\footnotetext{
* Manuscript received July 20, 2012; revised August 21, 2012.

Corresponding author. Tel.: +86-18610280438; E-mail address: wutao_victory@126.com.
} 
In order to provide a visual image evaluation on integrated online data and a more convenient data maintenance system for dispatch personnel to catch power system security and stability method, this paper proposes an online data assessment system concept based on E format, which combines online offline data matching, online offline parameter estimation and online data adjustment method to realize a practical real-time analysis on power system [7]-[10].

\section{Online/Offline Data Modeling Match}

Online data which contains power system structure, equipment parameters and operating information, can describe the power system network operation status information at a point accurately. But it is impossible to analyze the transient state of the whole network because of lacking of transient parameters. Offline data contains a relatively complete model of a typical grid structure, grid equipment and parameter data to provide a detailed, in-depth analysis of the physical characteristics of the power system, but cannot reflect real-time information of the power system operation currently[6]. Therefore, the online calculation data and offline data is described as the same power system but for different purposes, and the modeling carrier and the modeling process is relatively independent, which forms two sets of data to describe the power system from a different perspective. In fact, it is a different view of the real grid system [7]-[10].

\subsection{Online/offline data matching objective}

According to the characteristics of online data and offline data[11],[12], it needs to match the online and offline model data. Data after integration and match has the following objectives:

- Integrated data should maintain the data's completeness, including complete details of the grid model and parameters;

- The power flow of integrated data should be correspondence with online data;

- The integrated data should be automatically adapted to the addition, deletion and modification of offline data;

- The integrated data should be automatically adapted to the topology changes of online power system;

- The integrated data should provide improved methods of data viewing, comparison, calibration and correction;

\subsection{Online/offline data matching objective}

Online data model reflects the online real-time model. Sometimes, the online data model does not match with the actual situation for the delay of maintenance and update. However, offline data model can reflect the future situation of power system and contain the present model of power system. Using online/offline data model matching, online data model can keep real-time detection so that it could analyse the situations of unmatched models to realize real-time stability[13-15].

- Dynamic equipment mapping regards substations as basic mapping units. In the calculation data of power grid, the substations are located in the middle layer of the structure of power grid. For numbers of substations is relatively limited, it is easy to maintain substation mapping between different data. Meanwhile, the building of grid equipment mapping is simplified greatly after the substation mapping is formed.

- Dynamic mapping is realized by employing technology based on standard naming of substations and equipment.

- The complex mapping relationship between equivalent equipment and equivalent grid is realized based on equivalent attributes of the connecting equipment (lines or transformers) and other equipment. The modeling of offline data and online data do not match: in some cases online data only contains equivalent-measured information of substations' outgoing line; in other cases when some substations run ahead of schedule in the actual network, online calculation data has detailed substation model while offline data is relatively simple. Under this circumstance, it is necessary to build complex 
mapping relationship so as to ensure an accurate and complete reflection of online measurement of equivalent equipment and equivalent network in power flow calculation.

- Check structural deviation of the grid topology and load equivalent deviation caused by the modeling scope and level of information detail, by looking at the substation, generator, AC line, DC line, load, two-winding transformer, three winding transformer, each online/offline automatically mapping situation of circuit reactance shunt capacitance. Then it provides the connected components of online station nearby for non-match station, which could use connecting elements conveniently to find the corresponding station in the offline station.

\section{Online/Offline Parameter Estimation}

\subsection{Online/offline parameter estimation design}

As we all know, parameter estimate result is an important factor to reflect the power flow predictability. At present, we use the measured data of SCADA to estimate parameters. Paper [16] proposed an extended method of parameter estimation, which is called suspicious branch flow compensation analysis method. However, according to this method, all the parameters must be estimated to get accuracy results on a practical scenario. If some parameters can be estimated, it may lead to unfilled rank and finally causes the failure of iterative calculation. Paper [16] proposes to add bad data identification links to parameter estimation and clear up some residual data which is greater than the target parameter estimates. It can decrease the augmented Jacobian matrix information and make the iterative calculation more convenient. The actual project, adding bad data identification, may lead to multiple local extremum issue. In the theoretical analysis, because the residuals of the relevant line is still great, removing bad data line often causes related parameters with great deviation in line measurement to be deleted and then leads to a prompt drop on objective optimization value. Although Newton's method has local optimality, but Newton's method is sensitive to initial value which is closely associated with measurement deviation. Above all, adding link to indentify bad data has made sense on system parameter estimation but with deficiencies.

This paper proposes a method which cross matches the online/offline data integration, power flow data from SCADA and state estimation data to analyze data problem and provide solutions and ideas. The whole method is shown in Fig. 1.

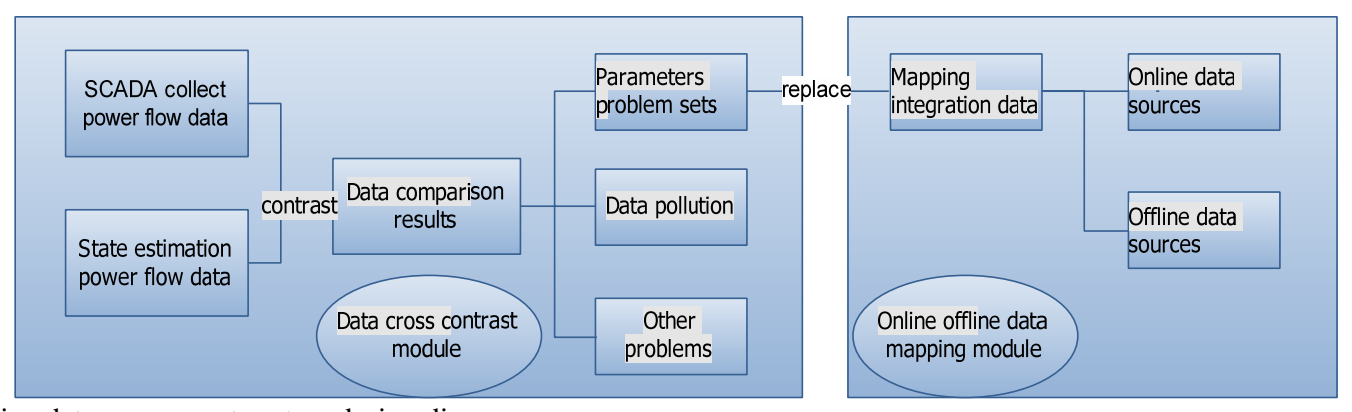

Fig. 1. Online data assessment system design diagram

- The whole system can be divided into data cross contrast module and online/offline data mapping module. In data cross contrast module, power flow data from SCADA is compared with that from state estimation. Firstly, it can verify the correctness of state estimation power flow. Secondly, it can find the cause of wrong data to improve the overall data quality.

- From the current operation of the system, the reason causing large deviation on parameters lies in the parameter problem and data pollution problem. In state estimation, the necrosis data will be cleared automatically but this may cause the data with large deviation is removed too. That will cause data pollution problem. Through the comparison of power flow data, bus voltage, and load etc, it is found that the main reason of parameters problem may be unreasonable state estimation or the data itself or any other factitious problem. 
- For parameters problem, we can contrast the online data with offline data by mapping data. In the situation that online/offline data updates timely, we could check and calibrate regional power, node voltage, generator and the mapping relationship. Then a set of integration data will be produced and will be used to replace some wrong data. Lastly calculate power flow and estimated state with this new data to get a set of good data results.

\subsection{Online/offline parameter estimation methods}

Parameter comparison is based on the Tabu's searching parameters which collect state estimation by SCADA [17-18]. This algorithm based on restrictive local search, which assigns the specified scope to estimate the optimized parameters solutions method (weighted residual square solution) as a parameter estimation results. The following is a basic description of the method.

- Make program code for estimate parameters which includes data before and after decimal point.

- Invert the experimental value gradually, whose quantity can be set in manual control level.

- Limit the search direction, which makes initial screening on experimental value. Select the experimental value selection principle and to establish a taboo list. Delete some experimental value which is beyond the boundary until a good experimental value is found.

- Set the target that is the desired level. Repeat steps 1-3 until estimation on the parameter results which comply with the convergence condition is done. It is sure that the optimized objective should be under the overall convergence of the desired level. The whole process is shown in Fig. 2.

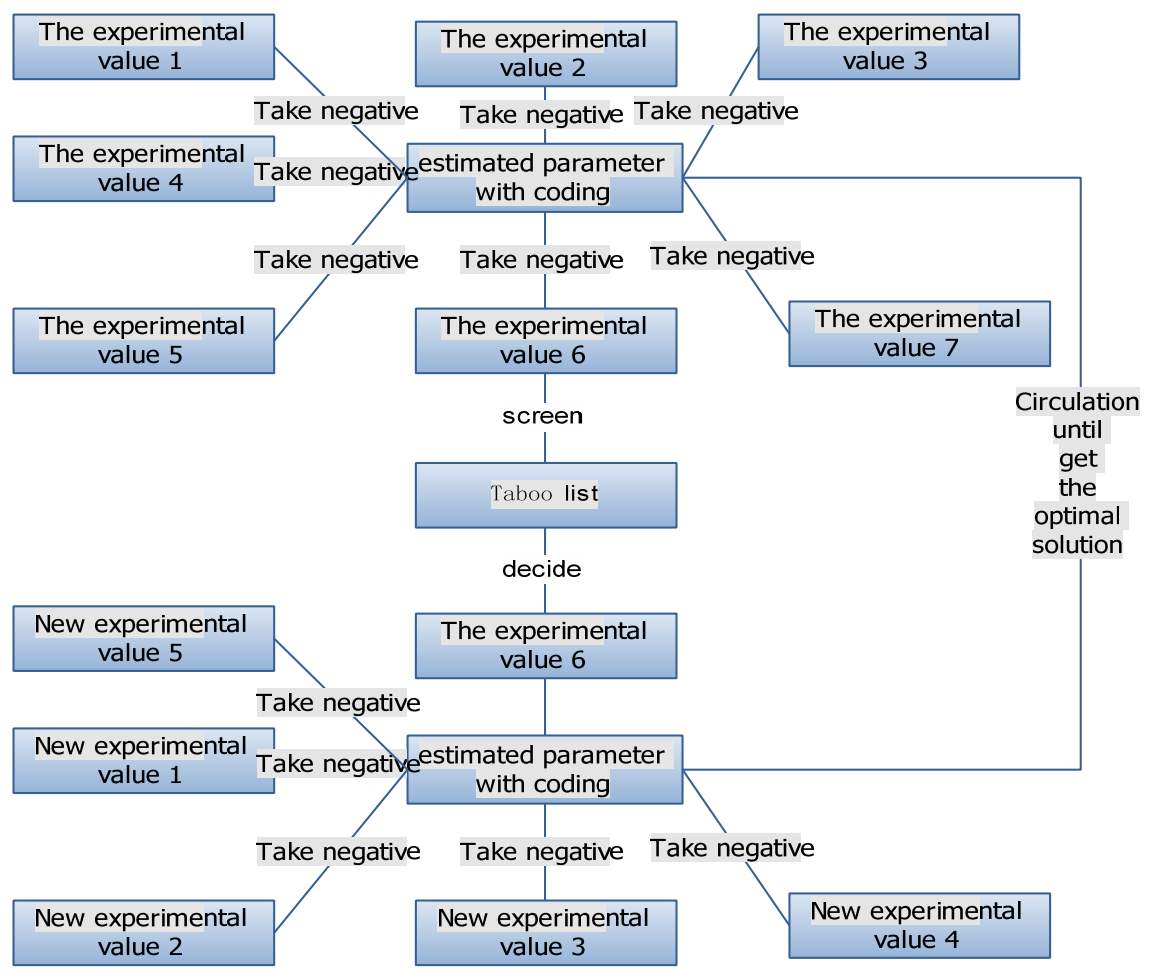

Fig. 2. Online/offline parameter estimation method.

\section{Online Data Adjustment}

\subsection{Step of online data adjustment}

To online data assessment, it must satisfy the dynamic stability calculation and convergence. So the adjustment on state estimation data is necessary. On one hand, the adjusted date can reflect the requirement of current system real-time operation; on the other hand it can satisfy the constraint of dynamic stability calculation. 


\subsection{Steps of online unbalance power flow adjustment}

By adjusting the online data nodes, it makes data satisfy the Kirchhoff's law and the branch power flow. If the power flow is not convergent, it is rational to distribute unbalance power flow to load in the adjacent station. Firstly, calculate the unbalanced power. Then share unbalanced load to adjacent load according to the load dispatching coefficient, by searching load of adjacent station and comparing the transformer capacity and corresponding load power flow margin.

\subsection{Step of online generator adjustment}

If the bus voltage generator is higher than 1.05 or lower than 0.98 , the ratio of transformer will change. All generators must be mapped with offline generators. Unmapped unit should be changed to constant impedance load except necessary outer equivalent generator. The upper limit and lower limit of active power and reactive power should use offline value.

\subsection{Step of online generator adjustment}

If the active power or reactive power of one of loads is less than 0 , all load power flow of this bus should be added. If it is still less than 0 , the load type should be changed to Constant impedance.

\section{Example}

Regarding the DC bipolar latching fault, we reproduced the failure dynamic process based on online data, and compared online data with PMU measured results and examine the simulation quality of the online data. Online data is extracted from the dynamic warning system in north and central China network data. Take pre-failure time as the basis of power flow data.

After assessment of the evaluation system of online data, we have adjusted the dynamic model parameters. Fig. 3 shows the simulation results after data adjustment and PMU measured results. It is clear that the simulate results after data correction are much closer to the real situation, which testifies the necessity of online data assessment.

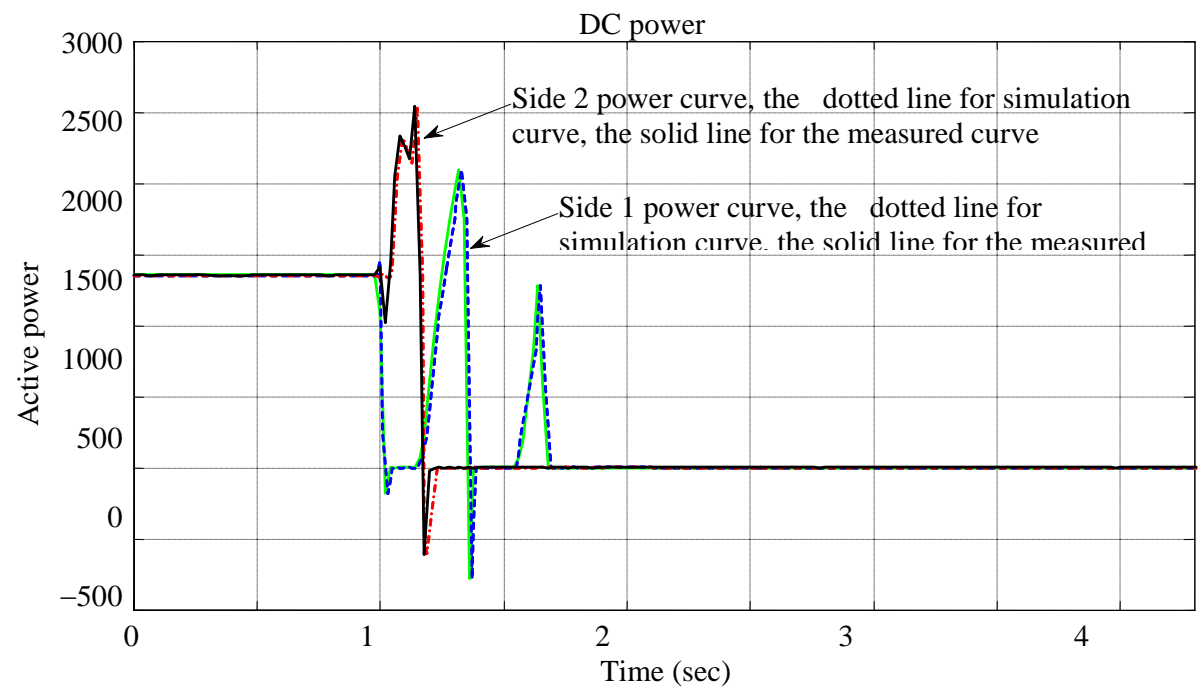

Fig. 3. DC bipolar latching fault simulation.

Table 1. DC bipolar latching fault simulation results

\begin{tabular}{llccl}
\hline Prony analysis UHV power & $\begin{array}{l}\text { Fluctuation } \\
(10000 \mathrm{~kW})\end{array}$ & $\begin{array}{c}\text { Amplitude } \\
(10000 \mathrm{~kW})\end{array}$ & $\begin{array}{c}\text { frequency } \\
(\mathrm{Hz})\end{array}$ & Deboost (\%) \\
\hline Original online data & 44.6 & 62 & 0.074 & 10.5 \\
\hline Improved online data & 68.5 & 51 & 0.193 & 12.46 \\
\hline PMU measured & 94.8 & 63.5 & 0.155 & 11 \\
\hline
\end{tabular}




\section{Conclusion}

With the rapid development of power system theory, computer technology and multimedia technology, it provides multiple solutions for the rapid development of power system. The gradual formation of the Sanhua power systems and the promotion of smart grid put forward more serious challenges on the safe and stable operation of power grid. Under such circumstance, it is significant to give assessment and decision on the online safety and stability of power grid through applying all kinds of resources. But the existing data fails to provide a grid model with complete information to reflect the real-time operational information for online security and stability assessment and decision-making. So it is important to integrate online operation data with offline data analysis. High-quality data reflects the real grid situation more authentically and specifically. It helps the dispatcher to make an accurate judgment on the possible risks of the current system and keeps a healthy and stable operation of the power system in a long term. This paper proposes the principles of quantitative assessment on integrated data quality. It can analyze the integration results from multiple angles and levels, and its friendly visualized interface, convenient operation and maintenance features can greatly reduce the intensity and workload of the dispatchers.

\section{References}

[1] Yin Y, Guo J, Zhao J, et al. Preliminary analysis of large scale blackout in interconnected North America power grid on August 14 and lessons to be drawn. Power System Technology, 2003; 27(10):1-5 (in Chinese) .

[2] Liu Y, Xie K. Analysis on blackout of interconnected North America power grid occurred on Aug 14, 2003 from the viewpoint of power system dispatching. Power System Technology, 2004; 28(8):10-15 (in Chinese).

[3] $\mathrm{Hu} \mathrm{X}$. Rethinking and enlightenment of large scope blackout interconnected North America power grid. Power System Technology, 2003; 27(9):T2-T6 (in Chinese).

[4] Zheng C, Hou J, et al. Functional design and implementation of online dynamic security assessment and early warning system. Power System Technology, 2010; 34(3):55-59 (in Chinese).

[5] Xin Y, Tao H, Li Y, et al. E language for electric power system model description. Automation of Electric Power Systems, 2006; 30(10).

[6] Yang Y, Tao H, Li Y, et al. Research and practice of integration of information in power dispatching center. Relay, 2007; 35(17):37-40.

[7] Wang J, Zhang C. Survey on power information resources integration methods. Power System Technology, 2006; 30(9): 83-87.

[8] Wang J. An integration solution of power information resources based on grid technology. Automation of Electric Power Systems, 2006; 30(20):84-87.

[9] Shi J, Li Y, Pen Q. The think of power system dispatching data integration program. Power System Technology, 2006; 30(10).

[10] Guo Qi. Research on Power System Security Warning and Coordination Prevention Control. Beijing: Tsinghua University, 2005.

[11] Yan J, Yu Z, Tian F, et al. Dynamic security assessment \& early warning system of power system. Proceedings of the CSEE, 2008; 28(34):87-93 (in Chinese) .

[12] Zhou X, Guo J, Sun Y. Research on Large Interconnected Power System Reliability. Beijing: Tsinghua University, 2008:184196.

[13] Yang Y, Zhang D. Study on the architecture of security and defense system of large-scale power grid. Power System Technology, 2004; 28(9):23-27 (in Chinese) .

[14] Zhao L, Guo Q, Tan Q. Ultra-high voltage synchronization grid stability analysis. Proceedings of the Chinese Society for Electrical Engineering, 2008; 28(34):47-51.

[15] Zhao L, Guo Q, Tan Q, et al. Analysis on stability characteristics of UHV synchronized power grid. Proceedings of the CSEE, 2008; 28(34):47-51 (in Chinese).

[16] Glover F, Laguna M. Tabu Search. Basel: Science Publisher, 1993:1-16.

[17] Glover F. Tabu Search-Part I. ORSA Journal on Computing, 1989; 1(3):190-206.

[18] Glover F. Tabu Search-Part II. ORSA Journal on Computing, 1990; 2(1):4-32. 